Bolyai Society - Springer-Verlag

\title{
A LOWER BOUND FOR THE TRANSLATIVE KISSING NUMBERS OF SIMPLICES
}

\author{
ISTVÁN TALATA
}

Received May 18, 1998

The translative kissing number $H(K)$ of a $d$-dimensional convex body $K$ is the maximum number of mutually non-overlapping translates of $K$ that can be arranged so that all touch $K$. In this paper we show that $H\left(S_{d}\right) \geq 1.13488^{(1-o(1)) d}$ holds for any $d$-dimensional simplex $S_{d}(d \geq 1)$. We also prove similar inequalities for some, more general classes of convex bodies.

\section{Introduction}

First we recall some standard definitions. By a d-dimensional convex body we mean a compact convex subset of $R^{d}$ with non-empty interior. Two subsets of $R^{d}$ with non-empty interiors are non-overlapping if they have no common interior point, and we say that they touch each other if they are non-overlapping and their intersection is non-empty. Denote by $H(K)$ the translative kissing number of a $d$-dimensional convex body $K$, which is defined as the maximum number of mutually non-overlapping translates of $K$ that can be arranged so that all touch $K . H(K)$ is often called the Hadwiger number of $K$ as well.

By a result of Swinnerton-Dyer [18] it follows that $H(K) \geq d^{2}+d$ holds for every $d$-dimensional convex body $K(d \geq 1)$. Recently, Talata [19] improved on this lower bound for sufficiently large values of $d$, showing that there exists an absolute constant $c>0$ such that $H(K) \geq 2^{c d}$ for every $d$-dimensional

Mathematics Subject Classification (1991): 52C17, 05D05

The work was partially supported by the Hungarian National Science Foundation, grant no. A-221/95 
convex body $K$. Combining this result with the general upper bound $H(K) \leq$ $3^{d}-1$ of Hadwiger [12], it turns out that the order of magnitude of $H(K)$ is exponential in the dimension of $K$ for every convex body $K$.

Since in [19] only the existence of an exponential lower bound is proved, so it is a natural problem to find explicit lower bounds of this kind for the translative kissing numbers, which are valid at least for some classes of convex bodies. There are two such previously known bounds. Groemer [10] proved that $H(K)=3^{d}-1$ if and only if $K$ is a paralleletope. In the case of Euclidean balls, the exponential lower bound $H(K) \geq(2 / \sqrt{3}-o(1))^{d}$ was found by Shannon [16] and Wyner [22].

In this paper we give an explicit exponential lower bound for the translative kissing numbers of simplices. We note that their exact values are known only in two and three dimensions (Grünbaum [11], Talata [20]). Furthermore, we generalize the obtained lower bound for $d$-orthoplexes. This important class of convex polytopes occurs in the Delaunay tilings corresponding to some well-known lattice packings of spheres (see Bezdek et al. [2], Conway and Sloane [4], [5]). We also extend a result of Talata [19] for a special class of non-symmetric convex bodies, proving an exponential lower bound for a quantity analogous to the translative kissing numbers defined for pairs of convex bodies. Finally, we show an inequality which strongly supports the conjecture that $H\left(S_{d}\right) \leq(3 / 2+o(1))^{d}$.

The common feature of the above mentioned statements that we are going to prove is that although they are purely geometric, their proofs share on ideas with which we are able to reduce them to suitable combinatorial problems on extremal sets.

For additional related results and references on this topic, see Bezdek [1], Bezdek et al. [3], Talata [21], and the survey papers by Fejes Tóth [7], Fejes Tóth and Kuperberg [8], and Zong [23].

\section{Results}

Our main result is the following exponential lower bound for the translative kissing numbers of simplices.

Theorem 1. Let $d$ be a positive integer, and let $S_{d}$ be a $d$-dimensional simplex. Then

$$
H\left(S_{d}\right) \geq 1.13488^{(1-o(1)) d}
$$

holds.

In the following we generalize Theorem 1 for $d$-orthoplexes. These polytopes play an important role in the theory of lattice packings of spheres. A 
$d$-orthoplex is a $d$-polytope which is congruent to a subset of $R^{d+1}$ that can be obtained as the intersection of a $(d+1)$-cube with a hyperplane orthogonal to a main diagonal of the cube. Many of these convex polytopes occur in the Delaunay tilings corresponding to some well-known lattice packings of spheres (see Bezdek et al. [2], Conway and Sloane [4], [5]). Since the translative kissing numbers are affine invariant quantities, and a regular $d$-simplex is also a $d$-orthoplex, thus the following theorem is a direct generalization of the previous one.

Theorem 2. Let $d$ be a positive integer, and let $P$ be a $d$-orthoplex. Then

$$
H(P) \geq 1.13488^{(1-o(1)) d}
$$

holds.

Before we formulate another generalization of Theorem 1 (but in this case with a bit smaller lower bound), we recall some notations. We use the standard notation $A+B$ for the set $\left\{a+b \in R^{d} \mid a \in A, b \in B\right\}$ for arbitrary $A, B \subseteq R^{d}$, and $A+v$ for the set $A+\{v\}$ for any $v \in R^{d}$. We write $A-B$ for short for the set $A+(-1) B$.

If $K$ and $L$ are $d$-dimensional convex bodies, then let us denote by $H(K, L)$ the maximum number of mutually non-overlapping translates of $L$ which touch $K$. From Corollary 4 of [19] it follows that there exists an absolute constant $c>0$ such that

$$
\max (H(K, L), H(L, K)) \geq 2^{c d}
$$

for every pair $(K, L)$ of centrally symmetric $d$-dimensional convex bodies. We now extend this result for the special class $\left\{\alpha S_{d}-\beta S_{d} \mid \alpha, \beta \in R\right\}$ of not necessarily symmetric convex bodies.

Theorem 3. Let $d$ be a positive integer, and let $S_{d}$ be a $d$-dimensional simplex. Let $\alpha, \beta, \gamma, \delta \geq 0$ be given with $\alpha+\beta \geq \gamma+\delta>0$. Let $K=\alpha S_{d}-\beta S_{d}$, $L=\gamma S_{d}-\delta S_{d}$. Then

$$
H(K, L) \geq 1.06744^{(1-o(1)) d}
$$

holds.

From the proof of Theorem 1 (see in Section 3) it turns out that there we use very special configurations to get the lower bound. This gives the reason for the following definition. For an $O$-symmetric convex polytope $P$ we define the centered translative kissing number $H_{c}(P)$ of $P$ as the maximum number of mutually non-overlapping translates $P_{1}, P_{2}, \ldots, P_{m}$ of $P$ which touch $P$ so that $P \cap P_{i}$ contains a common baricenter (center of mass) of some equal 
dimensional faces of $P$ and $P_{i}$, respectively, for every $1 \leq i \leq m$ (we denoted by $o$ the origin of $R^{d}$ ). We note that $H(P) \geq H_{c}(P)$ follows directly from the definitions. In the proof of Theorem 1 in fact we prove the lower bound for $H_{c}\left(C_{d}\right)$, where $C_{d}=\frac{1}{2}\left(S_{d}-S_{d}\right)$. Since $H\left(S_{d}\right)=H\left(C_{d}\right)$ (see below), this implies Theorem 1. To see how good our method is, we prove the following.

Theorem 4. Let $d$ be a positive integer, let $S_{d}$ be a $d$-dimensional simplex and $C_{d}=\frac{1}{2}\left(S_{d}-S_{d}\right)$. Then

$$
H_{c}\left(C_{d}\right) \leq\left(\frac{3}{2}\right)^{(1+o(1)) d}
$$

holds.

For a further clarification of the close relation between $H(P)$ and $H_{c}\left(\frac{1}{2}(P-P)\right)$ for a polytope $P$, we recall some definitions and concepts which will be needed in the proofs as well.

If $K$ is an $o$-symmetric $d$-dimensional convex body, then a norm $\|\cdot\|_{K}$ can be defined on $R^{d}$ for which $\|v\|_{K}=\frac{\|v\|}{\|w\|}$ for every $v \in R^{d}$, where $\|\cdot\|$ denotes the usual Euclidean norm, and $w$ is a vector parallel to $v$ and having endpoint on the boundary $\partial K$ of $K$. The metric derived from this norm is called the Minkowski metric determined by $K$. A set $S$ is called 1-discrete in the Minkowski metric determined by $K$ if $\left\|s_{1}-s_{2}\right\|_{K} \geq 1$ for any $s_{1}, s_{2} \in S$. If the Minkowski metric is fixed, then we call $S$ 1-discrete for short.

By Minkowski [14] (also see [15], [20]), for a convex body $K$ the quantity $H(K)$ is equal to the maximum cardinality of 1-discrete subsets of $\partial\left(\frac{1}{2}(K-K)\right)$ in the Minkowski metric determined by $\frac{1}{2}(K-K)$. This clearly implies that $H(K)=H\left(\frac{1}{2}(K-K)\right)$. Similarly it can be seen that for an $o$-symmetric polytope $P$ we have that $H_{c}(P)$ is equal to the maximum cardinality of 1-discrete subsets of $\mathcal{B}(P)$ in the Minkowski metric determined by $P$. Here we used the notation $\mathcal{B}(P)$ for the set of baricenters of the faces of $P$.

These facts strengthen a conjecture that the order of magnitudes of $H(P)$ and $H_{c}\left(\frac{1}{2}(P-P)\right)$ are the same if $P$ is a convex polytope for which $\frac{1}{2}(P-P)$ is symmetric enough. In the following we formulate this conjecture in two special cases. The first conjecture is the counterpart of Theorem 4 while the second one is the counterpart of the already mentioned lower bound $H(K) \geq$ $2^{c d}$ of [19] restricted to zonotopes. A zonotope is a convex polytope whose faces (of any dimensions) are centrally symmetric (for more information about zonotopes, see [9]). 
Conjecture 5. Let $d$ be a positive integer, and let $S_{d}$ be a $d$-dimensional simplex. Then

$$
H\left(S_{d}\right) \leq\left(\frac{3}{2}\right)^{(1+o(1)) d}
$$

holds.

Conjecture 6. There exists an absolute constant $c_{0}>0$ such that

$$
H_{c}(P) \geq 2^{c_{0} d}
$$

holds for any positive integer $d$ and any $d$-dimensional zonotope $P$.

\section{Proofs}

In the proofs we will consider $d$-dimensional convex bodies embedded into parallel hyperplanes of $R^{d+1}$. We note that then for two such convex bodies $K_{1}, K_{2}$, and for any vector $v \in R^{d+1}, K_{1}+\left(K_{2}+v\right)$ will be a translate of the $d$-dimensional convex body $K_{1}+K_{2}$, both of them are contained in some hyperplanes of $R^{d+1}$. If a $d$-dimensional convex body $K$ is contained in a hyperplane, and it is symmetric to the origin $o$ of $R^{d+1}$, then the Minkowski metric determined by $K$ can be defined on that hyperplane analogously to the case $K \subseteq R^{d}$. The quantities $H(K)$ and $H\left(K_{1}, K_{2}\right)$ can also be defined in this case with the restriction that all the translates of $K$ [resp. $K_{2}$ ] should lie in the same hyperplane as $K$ [resp. $\left.K_{1}\right]$.

We now introduce some notation. If $a \in R$, then denote by $[a]_{+}$the positive part of $a$ : $[a]_{+}=a$ if $a>0$, and $[a]_{+}=0$ if $a \leq 0$. Similarly, denote by $[a]_{-}$the negative part of $a$, that is $[a]_{-}=-a+[a]_{+}$. For a finite set $S$ we use the notation $|S|$ for the number of its elements. For $x \in R^{d+1}$ we denote by $x_{i}$ the $i$-th coordinate of $x$. For an arbitrary $r \in R$ we introduce the notation

$$
H_{r}=\left\{x \in R^{d+1} \mid \sum_{i=1}^{d+1} x_{i}=r\right\} .
$$

Then $H_{r}$ is an affine hyperplane in $R^{d+1}$, thus $H_{r} \cong R^{d}$. Since all the quantities in the theorems are affine invariant, therefore in the proofs we may assume that $S_{d}=\operatorname{conv}\left(e_{i} \mid 1 \leq i \leq d+1\right)$, where $\left\{e_{i}\right\}_{i=1}^{d+1} \subseteq R^{d+1}$ is given as

$$
\begin{aligned}
e_{1} & =(1,0, \ldots, 0) \\
e_{2} & =(0,1, \ldots, 0) \\
\vdots & \\
e_{d+1} & =(0,0, \ldots, 1) .
\end{aligned}
$$


Thus $S_{d} \subseteq H_{1}$. Let $C_{d}=\frac{1}{2}\left(S_{d}-S_{d}\right)$, and $C r_{d+1}=\operatorname{conv}\left( \pm e_{i} \mid 1 \leq i \leq d+1\right)$. $C r_{d+1}$ is called the crosspolytope of $R^{d+1}$. It is clear that $C_{d}=C r_{d+1} \cap H_{0}$.

Proof of Theorem 1. Let $k, l \geq 1, k+l \leq d+1$. Define

$$
\begin{array}{r}
F_{k, l}=\left\{x \in H_{0} \mid x_{i} \geq 0 \forall i \leq k, x_{i} \leq 0 \forall k<i \leq k+l, x_{i}=0 \forall i>k+l,\right. \\
\left.\sum_{i=1}^{d+1}\left[x_{i}\right]_{+}=\sum_{i=1}^{d+1}\left[x_{i}\right]_{-}=\frac{1}{2}\right\} .
\end{array}
$$

It is easy to see that $F_{k, l}$ is a $(k+l-2)$-dimensional face of $C_{d}$. Let $\mathcal{F}_{k, l}=$ $\left\{\tau\left(F_{k, l}\right) \mid \tau \in \mathcal{T}_{d+1}\right\}$, where $\mathcal{T}_{d+1}$ denotes the collection of all transformations of $R^{d+1}$ which are formed by coordinate permutations. For $0 \leq r \leq d-1$, let $\mathcal{F}_{r}=\bigcup_{k+l=r+2} \mathcal{F}_{k, l}$. It is clear that $\mathcal{F}_{r}$ is the collection of all $r$-dimensional faces of $C_{d}$. Define a point $b_{k, l} \in F_{k, l}$ as

$$
b_{k, l}=\left(\frac{1}{2 k}, \ldots, \frac{1}{2 k},-\frac{1}{2 l}, \ldots,-\frac{1}{2 l}, 0, \ldots, 0\right) .
$$

Then $b_{k, l}$ is the baricenter (the center of mass) of $F_{k, l}$. Let $\mathcal{B}_{k, l}=\left\{\tau\left(b_{k, l}\right) \mid\right.$ $\left.\tau \in \mathcal{T}_{d+1}\right\}$. For $0 \leq r \leq d-1$, let $\mathcal{B}_{r}=\bigcup_{k+l=r+2} \mathcal{B}_{k, l}$. Then $\mathcal{B}_{r}$ is the collection of the baricenters of all $r$-dimensional faces of $C_{d}$. Let $\mathcal{B}=\bigcup_{r=0}^{d-1} B_{r}$.

We have $H\left(S_{d}\right)=H\left(C_{d}\right) \geq H_{c}\left(C_{d}\right)$. Define $H_{k, l}\left(C_{d}\right)$ as the maximum cardinality of 1-discrete subsets of $\mathcal{B}_{k, l}$ in the Minkowski metric determined by $C_{d}$. Since $k+l \leq d+1$, so we have that

$$
\max _{k, l} H_{k, l}\left(C_{d}\right) \leq H_{c}\left(C_{d}\right) \leq \frac{d^{2}+d}{2} \max _{k, l} H_{k, l}\left(C_{d}\right) .
$$

Let $x, y \in H_{0}$. By $C_{d}=C r_{d+1} \cap H_{0}$ we get that $\|x-y\|_{C_{d}} \geq 1$ if and only if $\sum_{i=1}^{d+1}\left[x_{i}-y_{i}\right]_{+} \geq 1 / 2$.

Let $\left\{q_{i}\right\}_{i=1}^{m} \subseteq \mathcal{B}_{k, l}$. Denote by $q_{i, j}$ the $j$-th coordinate of $q_{i}(1 \leq i \leq m)$. Let $X_{d+1}=\{1,2, \ldots, d+1\}$. We define two subsets of $X_{d+1}$ for every $q_{i}$ as follows:

$$
\begin{aligned}
& A_{i}=\left\{n \in X_{d+1} \mid q_{i, n}>0\right\}, \\
& B_{i}=\left\{n \in X_{d+1} \mid q_{i, n}<0\right\} .
\end{aligned}
$$

Then for every $i$ we have

$$
\left\{\begin{array}{l}
\left|A_{i}\right|=k, \\
\left|B_{i}\right|=l, \\
A_{i} \cap B_{i}=\emptyset .
\end{array}\right.
$$


It is easy to check that $\left\|q_{i}-q_{j}\right\|_{C_{d}} \geq 1$ if and only if

$$
\frac{\left|A_{i} \cap A_{j}\right|}{k}+\frac{\left|B_{i} \cap B_{j}\right|}{l} \leq 1 .
$$

Consequently we have the following.

Lemma 7. $H_{k, l}\left(C_{d}\right)$ is the maximum number $m$ for which there exists a collection $\left\{\left(A_{i}, B_{i}\right)\right\}_{i=1}^{m}$ of pairs of subsets of $X_{d+1}$ so that (3) and (4) hold for every $i \neq j$.

Consider now the case $l=k$. Then there are

$$
f(d, k)=\left(\begin{array}{c}
d+1 \\
2 k
\end{array}\right)\left(\begin{array}{c}
2 k \\
k
\end{array}\right)
$$

different pairs $\left(A_{i}, B_{i}\right)$ for which $(3)$ holds. On the other hand, for such a fixed pair $\left(A_{i_{0}}, B_{i_{0}}\right)$ we have

$$
g(d, k)=\sum_{\substack{k_{1}+l_{2} \leq k \\ l_{1}+k_{2} \leq k \\ k_{1}+l_{1}>k}} h\left(d, k, k_{1}, k_{2}, l_{1}, l_{2}\right)
$$

different pairs $\left(A_{i}, B_{i}\right)$ for which (3) holds but (4) does not, where we used the notation

$$
\begin{aligned}
& h\left(d, k, k_{1}, k_{2}, l_{1}, l_{2}\right)= \\
& \quad C\left(k ; k_{1}, k_{2}\right) C\left(k ; l_{1}, l_{2}\right) C\left(d-2 k+1 ; k-k_{1}-l_{2}, k-l_{1}-k_{2}\right) .
\end{aligned}
$$

Here we denoted by $C(u ; v, w)$ the multinomial coefficient

$$
\frac{u !}{v ! w !(u-v-w) !} .
$$

With this notation we get $H_{k, k}\left(C_{d}\right) \geq f(d, k) / g(d, k)$. Denote by $h(d, k)$ the largest term of the sum in (6). Then we have that $g(d, k) \leq(d+1)^{4} h(d, k)$. This implies

$$
H_{k, k}\left(C_{d}\right) \geq(f(d, k) / h(d, k))(1-o(1))^{d} .
$$

To finish the proof, by (1) we need only to calculate the order of magnitude of the expression

$$
F(d)=\max _{k} \frac{f(d, k)}{h(d, k)}=\max _{k} \min _{k_{1}, k_{2}, l_{1}, l_{2}} \frac{f(d, k)}{h\left(d, k, k_{1}, k_{2}, l_{1}, l_{2}\right)},
$$

with the same restrictions in the minimum for $k_{i}, l_{i}(i=1,2)$ as in (6). 


\section{Lemma 8.}

$$
\lim _{d \rightarrow \infty}(F(d))^{1 / d}=G\left(b_{0}, z_{0}\right) \approx 1.13488449 \ldots,
$$

for $\left(b_{0}, z_{0}\right)$ where $b_{0} \approx 0.087378 \ldots$ is the unique real solution of the cubic equation

$$
b^{3}+5 b^{2}+11 b-1=0,
$$

and

$$
z_{0}=3-b_{0}+\frac{\left(1-b_{0}\right)^{2}}{2 b_{0}}
$$

where

$$
G(b, z)=z\left(\frac{2^{b} E(b) E^{2}(1-b) E(b+z-3)}{8 E(z-2)}\right)^{1 / z},
$$

with the notation $E(x)=x^{x}$.

Proof of Lemma 8. Writing $a_{i}=\frac{k_{i}}{k}, b_{i}=\frac{l_{i}}{k}(i=1,2)$ and $z=\frac{d+1}{k}$, by (5)(8), using Stirling formula and elementary analysis to calculate the saddle point, we get that $a_{1}+o(1)=b_{1}+o(1)=\frac{1}{2}, b_{2}=a_{2}+o(1)$, and $z=3-$ $2 a_{2}+\frac{\left(1-2 a_{2}\right)^{2}}{4 a_{2}}+o(1)$. Setting $b=2 a_{2}$, we get $b^{3}+5 b^{2}+11 b-1+o(1)=$ 0 . Finally, substituting the (modulo $o(1)$ ) calculated value $b_{0}$ to $b$ in the obtained formulas, we get the value for the limit.

This completes the proof of Theorem 1 .

Proof of Theorem 2. Let $P$ be a $d$-orthoplex. By symmetry reasons, we may assume that $P=[0,1]^{d+1} \cap H_{r} \subseteq R^{d+1}$ for some $0<r \leq \frac{d}{2}$. Let $Q=\frac{1}{2 r}(P-P)$. Then $H(P)=H(Q)$. It is easy to see that $Q=C_{d} \bigcap\left[-\frac{1}{2 r}, \frac{1}{2 r}\right]^{d+1}$, so $\mathcal{B}_{k, l} \subseteq \mathcal{B}(Q)$ if $\min (k, l) \geq r$.

From $Q \subseteq C_{d}$ follows that $H_{k, l}\left(C_{d}\right) \leq H(Q)$ if $\min (k, l) \geq r$. On the other hand, by the proof of Theorem 1 (cf. Lemma 8) we have that $H_{k, k}\left(C_{d}\right) \geq$ $1.13488^{(1-o(1)) d}$ for $k=(d+1) / z_{0}+o(1)$, where $z_{0} \approx 7.678573 \ldots$ This shows that we are done with the proof if $(d+1) / z_{0} \geq r$. So, in the remaining part we may assume that $(d+1) / z_{0} \leq r$. Let $c=r /(d+1)$. Then $c \geq 1 / z_{0}>0.130232$. Choose $k=\lceil r\rceil$. Now, consider those coordinate permutations of $b_{k, k}$ whose nonzero coordinates are exactly the first $2 k$ coordinates. From the set formed by these points choose a maximal subset $\mathcal{A}$ for which $u+v \neq o$ for every $u, v \in \mathcal{A}$. Then $|\mathcal{A}|=\frac{1}{2}\left(\begin{array}{c}2 k \\ k\end{array}\right)=4^{(1-o(1)) c d} \geq 1.19786^{(1-o(1)) d}$. Finally, observe that $\mathcal{A} \subseteq \mathcal{B}_{k, k}$, and for every $u, v \in \mathcal{A}$ there is a coordinate of $u-v$ whose value is at least $\frac{1}{r}$, so $\mathcal{A}$ is 1 -discrete in the metric determined by $Q$. Consequently, in this case we have $H(Q) \geq H_{c}(Q) \geq 1.19786^{(1-o(1)) d}$. 
Proof of Theorem 3. If $K_{1}, K_{2}$ are $d$-dimensional convex bodies and $K_{2}$ is $o$-symmetric, then denote by $M\left(K_{1}, K_{2}\right)$ the maximum cardinalty of 1-discrete subsets of $\partial K_{1}$ in the Minkowski metric determined by $K_{2}$. If $K_{1}, K_{2} \subseteq R^{d+1}$, but they lie on parallel hyperplanes, then denote by $M\left(K_{1}, K_{2}\right)$ the quantity $M\left(K_{1}^{\prime}, K_{2}\right)$, where $K_{1}^{\prime}$ is an arbitrary translate of $K_{1}$ which lies in the same hyperplane as $K_{2}$. This way we have $H\left(K_{1}, K_{2}\right)=$ $M\left(K_{1}-K_{2}, K_{2}-K_{2}\right)$.

Let $\lambda=\frac{\alpha+\gamma}{\alpha+\beta+\gamma+\delta}$, and $C_{d}^{\lambda}=\lambda S_{d}-(1-\lambda) S_{d}$. Then $0 \leq \lambda \leq 1$, and

$$
H(K, L)=M(K-L, L-L)=M\left(C_{d}^{\lambda}, \frac{2(\gamma+\delta)}{\alpha+\beta+\gamma+\delta} C_{d}\right) \geq M\left(C_{d}^{\lambda}, C_{d}\right)
$$

since $\frac{\gamma+\delta}{\alpha+\beta+\gamma+\delta} \geq \frac{1}{2}$. Define

$$
\begin{aligned}
F_{k, l}^{\lambda}=\left\{x \in H_{\lambda} \mid x_{i} \geq 0 \forall i \leq k, x_{i} \leq 0 \quad\right. & \forall k<i \leq k+l, x_{i}=0 \forall i>k+l, \\
& \left.\sum_{i=1}^{d+1}\left[x_{i}\right]_{+}=\lambda, \sum_{i=1}^{d+1}\left[x_{i}\right]_{-}=1-\lambda\right\} .
\end{aligned}
$$

Then $F_{k, l}^{\lambda}$ is a $(k+l-2)$-dimensional face of $C_{d}^{\lambda}$. Let $\mathcal{F}_{k, l}^{\lambda}=\left\{\tau\left(F_{k, l}^{\lambda}\right) \mid \tau \in \mathcal{T}_{d+1}\right\}$, and let $\mathcal{F}_{r}^{\lambda}=\bigcup_{k+l=r+2} \mathcal{F}_{k, l}^{\lambda}$ for $0 \leq r \leq d-1$. It is easy to see that $\mathcal{F}_{r}^{\lambda}$ is the collection of all $r$-dimensional faces of $C_{d}^{\lambda}$. Define a point $b_{k, l}^{\lambda} \in F_{k, l}$ as

$$
b_{k, l}^{\lambda}=\left(\frac{\lambda}{k}, \ldots, \frac{\lambda}{k},-\frac{1-\lambda}{l}, \ldots,-\frac{1-\lambda}{l}, 0, \ldots, 0\right) .
$$

Then $b_{k, l}^{\lambda}$ is the baricenter (the center of mass) of $F_{k, l}^{\lambda}$. Let $\mathcal{B}_{k, l}^{\lambda}=\left\{\tau\left(b_{k, l}^{\lambda}\right) \mid\right.$ $\left.\tau \in \mathcal{T}_{d+1}\right\}$. For $0 \leq r \leq d-1$, let $\mathcal{B}_{r}^{\lambda}=\bigcup_{k+l=r+2} \mathcal{B}_{k, l}^{\lambda}$. Then $\mathcal{B}_{r}^{\lambda}$ is the collection of the baricenters of all $r$-dimensional faces of $C_{d}^{\lambda}$. Let $\mathcal{B}^{\lambda}=\bigcup_{r=0}^{d-1} B_{r}^{\lambda}$.

Let $\left\{q_{i}\right\}_{i=1}^{m} \subseteq \mathcal{B}_{k, l}^{\lambda}$. Now, if we define the sets $A_{i}, B_{i}$ by (2), then we have (3). It is easy to check that $\left\|q_{i}-q_{j}\right\|_{C_{d}} \geq 1$ if and only if

$$
\lambda \frac{\left|A_{i} \cap A_{j}\right|}{k}+(1-\lambda) \frac{\left|B_{i} \cap B_{j}\right|}{l} \leq \frac{1}{2} .
$$

Consequently we have that $M\left(C_{d}^{\lambda}, C_{d}\right) \geq m$ if there exists a collection $\left\{\left(A_{i}, B_{i}\right)\right\}_{i=1}^{m}$ of pairs of subsets of $X_{d+1}$ so that (3) and (9) hold for every $1 \leq i, j \leq m$.

Let $1 \leq s \leq d-1$ be an integer number. Set $k=\lceil\lambda s\rceil, l=\lceil(1-\lambda) s\rceil$ and $r=k+l$. Then (9) holds if

$$
\left|A_{i} \cap A_{j}\right|+\left|B_{i} \cap B_{j}\right| \leq \frac{s}{2} .
$$


Let $Z_{i}=A_{i} \cup B_{i}$ for every $1 \leq i \leq m$. Assume that $a_{i}<b_{i}$ for every $a_{i} \in A_{i}, b_{i} \in$ $B_{i}$. Then $Z_{i} \neq Z_{j}$ for every $i \neq j$, and $s \leq\left|Z_{i}\right|=r \leq s+2$. Furthermore,

$$
\left|Z_{i} \cap Z_{j}\right|<\frac{r}{2}
$$

implies (10). We note that if $Z_{i}=\left\{n_{i, j}\right\}_{j=1}^{r}$ with $n_{i, 1}<n_{i, 2}<\ldots<n_{i, r}$, then $A_{i}=\left\{n_{i, j}\right\}_{j=1}^{k}$ and $B_{i}=\left\{n_{i, j}\right\}_{j=k+1}^{r}$.

This shows that $M\left(C_{d}^{\lambda}, C_{d}\right) \geq m$ if there exists a collection $\left\{Z_{i}\right\}_{i=1}^{m}$ of $r$-element subsets of $X_{d+1}$ so that (11) holds for every $i \neq j$.

By a similar argument to the one in the proof of Theorem 1, calculating that how many $r$-element subsets are in $X_{d+1}$, and that for a fixed $Z_{i}$ how many $Z_{j}$ 's $(j \neq i)$ are for which (11) does not hold, we get that

$$
H\left(C_{d}^{\lambda}, C_{d}\right) \geq \frac{\left(\begin{array}{c}
d+1 \\
r
\end{array}\right)}{\sum_{t \geq r / 2}\left(\begin{array}{c}
r \\
t
\end{array}\right)\left(\begin{array}{c}
d+1-r \\
r-t
\end{array}\right)} .
$$

Let

$$
F_{1}(d)=\max _{r} \min _{t \geq r / 2}\left(\begin{array}{c}
d+1 \\
r
\end{array}\right) /\left(\left(\begin{array}{l}
r \\
t
\end{array}\right)\left(\begin{array}{c}
d+1-r \\
r-t
\end{array}\right)\right) .
$$

Then $H\left(C_{d}^{\lambda}, C_{d}\right) \geq F_{1}(d)(1-o(1))^{d}$.

Lemma 9.

$$
\lim _{d \rightarrow \infty}\left(F_{1}(d)\right)^{1 / d}=G_{1}\left(z_{0}\right) \approx 1.06744224 \ldots,
$$

for $z_{0} \approx 7.222262 \ldots$, where $z_{0}$ is the unique real solution of the cubic equation

$$
z^{3}-10 z^{2}+22 z-14=0,
$$

and

$$
G_{1}(z)=z\left(\frac{E\left(z-\frac{3}{2}\right)}{\sqrt{8} E(z-1)}\right)^{1 / z}
$$

with the notation $E(x)=x^{x}$.

Proof of Lemma 9. Writing $b=\frac{t}{r}$ and $z=\frac{d+1}{r}$ in (12), using Stirling formula and elementary analysis to calculate the saddle point, we get that $b=\frac{1}{2}+o(1)$, and $z^{3}-10 z^{2}+22 z-14+o(1)=0$. Finally, substituting the (modulo $o(1)$ ) calculated value $z_{0}$ to $z$ in the obtained formulas, we get the value for the limit.

This completes the proof of Theorem 3 . 
Proof of Theorem 4. By Lemma 7 and (1) we have that there exist suitable $k, l$ and a collection $\left\{\left(A_{i}, B_{i}\right)\right\}_{i=1}^{m}$ of pairs of subsets of $X_{d+1}$ for which (3) and (4) hold for every $i \neq j$, such that $H_{c}\left(C_{d}\right) \leq(1+o(1))^{d} m$. Fix such a collection $\left\{\left(A_{i}, B_{i}\right)\right\}_{i=1}^{m}$.

Let $p=\left\lceil\frac{k}{2}\right\rceil, q=\left\lceil\frac{l}{2}\right\rceil+1, u=p+q$. Now, consider the $(U, V)$ pairs of subsets of $X_{d+1}$ for which

$$
\left\{\begin{array}{l}
|U|=p, \\
|V|=q, \\
U \cap V=\emptyset .
\end{array}\right.
$$

Then, by (3) there is no such a pair $(U, V)$ for which $U \subseteq A_{i} \cap A_{j}$ and $V \subseteq B_{i} \cap B_{j}$ for any $i \neq j$. Since the total number of $(U, V)$ pairs for which (13) holds is

$$
\left(\begin{array}{c}
d+1 \\
u
\end{array}\right)\left(\begin{array}{l}
u \\
p
\end{array}\right),
$$

and for a pair $\left(A_{i}, B_{i}\right)$ there exist

$$
\left(\begin{array}{l}
k \\
p
\end{array}\right)\left(\begin{array}{l}
l \\
q
\end{array}\right)=2^{(1-o(1)) u}
$$

pairs $(U, V)$ for which $U \subseteq A_{i}, V \subseteq B_{i}$, and (13) holds, thus we get

$$
m \leq \frac{\left(\begin{array}{c}
d+1 \\
u
\end{array}\right)\left(\begin{array}{l}
u \\
p
\end{array}\right)}{2^{(1-o(1)) u}} \leq F_{2}(d)=\max _{v, w} \frac{\left(\begin{array}{c}
d+1 \\
v
\end{array}\right)\left(\begin{array}{c}
v \\
w
\end{array}\right)}{2^{(1-o(1)) v}} .
$$

To find the order of magnitude of $F_{2}(d)$, write $c=\frac{d+1}{v}$ and $t=\frac{w}{v}$. Then, by Stirling formula and elementary analysis we get $t=\frac{1}{2}+o(1)$ and $c=\frac{1}{3}+o(1)$. Finally, substituting these obtained values to the calculated formulas, we get

$$
F_{2}(d)=\left(\frac{3}{2}\right)^{(1+o(1)) d} .
$$

This completes the proof of Theorem 4 .

\section{Concluding remarks}

Since $C_{d}=C r_{d+1} \cap H_{0}$, therefore Theorem 1 implies a lower bound of the same magnitude for the translative kissing numbers of crosspolytopes. Namely, we get $H\left(C r_{d}\right) \geq 1.13488^{(1-o(1)) d}$. This improves on the lower bound $H\left(C r_{d}\right) \geq$ $1.06739^{(1-o(\overline{1})) d}$ of Swanepoel [17]. 
A weaker version of Theorem 3 can be proved with simpler calculations using Turán numbers: Combining a result of Erdős and Spencer (p. 74 of [6]) and an inequality of Katona et al. [13] (also see as Theorem 13.1 in [6]), one can obtain

$$
H\left(C_{d}^{\lambda}, C_{d}\right) \geq \frac{\left(\begin{array}{c}
d+1 \\
\lfloor r / 2\rfloor
\end{array}\right)}{\left(\begin{array}{c}
r \\
\lfloor r / 2\rfloor
\end{array}\right)^{2}},
$$

which implies $H(K, L) \geq 1.0625^{(1-o(1)) d}$.

Lemma 7 can be reformulated in the language of hypergraphs. Let $\mathcal{H}_{k, l}$ be the hypergraph whose edges are formed by the collection $\left\{\left(A_{i}, B_{i}\right)\right\}_{i=1}^{n_{1}}$ of all pairs of subsets of $X_{d+1}$ for which (3) holds for every $1 \leq i \leq n_{1}$, and whose vertices are formed by the collection $\left\{\left(U_{j}, V_{j}\right)\right\}_{j=1}^{n_{2}}$ of all pairs of disjoint subsets of $X_{d+1}$ with

$$
\frac{\left|U_{j}\right|}{k}+\frac{\left|V_{j}\right|}{l}>1
$$

for every $1 \leq j \leq n_{2}$. If the incidence relation is defined by the rule that a vertex $\left(U_{j}, V_{j}\right)$ is adjacent to an edge $\left(A_{i}, B_{i}\right)$ if and only if $U_{j} \subseteq A_{i}$ and $V_{j} \subseteq B_{i}$, then we have that $H_{k, l}\left(C_{d}\right)$ is the maximum number of mutually disjoint edges of $\mathcal{H}_{k, l}$ (i.e. the size of maximal matchings of $\mathcal{H}_{k, l}$ ). It is worth further investigation if there exists a suitable subhypergraph of $\mathcal{H}_{k, l}$ for which the size of maximal matchings can be estimated more effectively so that it would imply an improvement of the lower bound of Theorem 1.

Acknowledgements. For the helpful discussions and encouragement, I am obliged to András Bezdek and Wlodzimierz Kuperberg.

\section{References}

[1] A. Bezdek: On the Hadwiger number of a starlike disk, Bolyai Soc. Math. Studies, (Intuitive Geometry, Budapest, 1995), 6 (1997), 237-245.

[2] A. Bezdek, K. Bezdek and R. Conelly: Finite and uniform stability of sphere packings, Discrete Comput. Geom., 20 (1998), 111-130.

[3] A. Bezdek, K. Kuperberg, and W. Kuperberg: Mutually contiguous translates of a plane disk, Duke Math. J., 78 (1995), 19-31.

[4] J. H. Conway and N. J. A. Sloane: Sphere Packings, Lattices and Groups, SpringerVerlag 1993.

[5] J. H. Conway and N. J. A. Slonne: The cell structures of certain lattices; Miscellanea Mathematica, (P. Hilton, F. Hirzebruch and R. Remmert eds.) Springer-Verlag 71-107, 1991.

[6] P. ERdős and J. Spencer: Probabilistic Methods in Combinatorics, Academic Press, New York, London, 1974. 
[7] G. Fejes Tóth: Packing and covering, in: Handbook of Discrete and Computational Geometry, (J. E. Goodman and J. O'Rourke eds.), CRC Press, Boca Raton, New York 19-41, 1997.

[8] G. Fejes Tóth and W. Kuperberg: Packing and covering with convex sets, in: Handbook of Convex Geometry, (P. M. Gruber and J. M. Wills eds.), North Holland, Amsterdam (1993), 799-860.

[9] P. Goodey and W. WeIL: Zonoids and generalisations, in: Handbook of Convex Geometry, (P. M. Gruber and J. M. Wills eds.), North Holland, Amsterdam (1993), $1297-1326$.

[10] H. Groemer: Abschätzungen für die Anzahl der konvexen Körper, die einen konvexen Körper berühren, Monatsh. Math., 65 (1961), 74-81.

[11] B. Grünbaum: On a conjecture of H. Hadwiger, Pacific J. Math., 11 (1961), 215219.

[12] H. Hadwiger: Über Treffenzahlen bei translationsgleichen Eikörpern, Arch. Math., 8 (1957), 212-213.

[13] G. Katona, T. Nemetz and M. Simonovits: On a graph-problem of Turán, (in Hungarian), Mat. Lapok, 15 (1964), 228-238.

[14] H. Minkowski: Dichteste gitterförmige Lagerung kongruenter Körper, Nachr. Ges. Wiss. Göttingen, (1904), 311-355.

[15] C. A. Rogers: Packing and Covering, Cambridge University Press, Cambridge (1964.

[16] C. E. Shannon: Probability of error for optimal codes in a Gaussian channel, Bell System Tech. J., 38 (1959), 611-656.

[17] K. J. Swanepoel: New lower bounds for the Hadwiger numbers of $\ell_{p}$ balls for $p<2$, Appl. Math. Lett., 1-6, to appear.

[18] H. P. F. Swinnerton-Dyer: Extremal lattices of convex bodies, Proc. Cambridge Philos. Soc., 49 (1953), 161-162.

[19] I. Talata: Exponential lower bound for the translative kissing numbers of $d$ dimensional convex bodies, Discrete Comput. Geom., 19 (1998), 447-455.

[20] I. Talata: The translative kissing number of tetrahedra is 18, Discrete Comput. Geom., 22 (1999), 231-248.

[21] I. Talata: On a lemma of Minkowski, Period. Math. Hungar., 36 (1998), 199-207.

[22] J. M. Wyner: Capabilities of bounded discrepancy decoding, Bell System Tech. J., 44 (1965), 1061-1122.

[23] C. Zong: The kissing numbers of convex bodies - A brief survey, Bull. London Math. Soc., 30 (1998), 1-10.

\section{István Talata}

Department of Mathematics, Auburn University, 218 Parker Hall, Auburn, AL 36849-5310, USA

and

Department of Mathematics, The University of Michigan, East Hall, 525 East University Avenue, Ann Arbor, MI 48109-1109, USA 